\title{
NOUVELLE
}

\section{Des mécanismes moléculaires conservés contrôlent la polarité cellulaire}

Sandrine Etienne-Manneville
Cnrs UMR 144, Institut Curie,

26, rue d'Ulm, 75248 Paris

Cedex 05, France.

sandrine. etienne-

manneville@curie.fr
La polarité cellulaire:

une propriété essentielle

au fonctionnement cellulaire

La polarité est une caractéristique fondamentale des cellules, qui régit de très nombreuses fonctions biologiques. Par exemple, la mise en place rapide de l'axe antéro-postérieur de l'œuf fécondé permet l'orientation du futur embryon. Au cours du développement, la polarité des cellules se traduit par une ségrégation asymétrique de facteurs de différenciation à des pôles opposés de la cellule, donnant naissance à des cellules filles différentes. Dans les cellules adultes différenciées, comme les cellules épithéliales, la face apicale est très nettement distincte de la face baso-latérale. Dans les neurones, des dendrites reçoivent les informations et un axone en permet la transmission. L'asymétrie des cellules et leur orientation peuvent aussi être modulées en réponse aux modifications de l'environnement. Ainsi, les cellules $T$ cytotoxiques se polarisent au contact de leur cellule cible, ce qui s'accompagne d'une orientation du cytosquelette des microtubules et donc du transport des vésicules d'exocytose vers la cellule cible [1]. Une force propulsive s'établit à l'avant d'une cellule qui migre, attirée par exemple par un chimio-attractant, alors que celle-ci se rétracte à l'arrière [2].

L'omniprésence de la polarité cellulaire pour des fonctions cellulaires extrêmement variées pose deux questions principales: quels molécules et mécanismes moléculaires contrôlent ce processus? Ces mécanismes et ces molécules sont- ils conservés dans les situations très diverses où la polarité cellulaire $(\rightarrow) \mathrm{m} / \mathrm{s}$ 2002, n०8-9, p. 142 se met en place?

Un complexe moléculaire très conservé : Cdc42, Par6 et les aPKC

De nombreuses molécules impliquées dans la mise en place de la polarité cellulaire ont été identifiées indépendamment dans les différents modèles d'étude facilement accessibles aux manipulations génétiques, comme la levure $S$. cerevisiae, le nématode $C$. elegans, la drosophile ou le xénope. Ce n'est que plus récemment que leur conservation a été reconnue.

II en est ainsi de la petite protéine $G$ Cdc42: son implication dans la polarité cellulaire a été initialement identifiée dans la levure où elle contrôle la polarisation au cours de la division cellulaire [3]. On sait depuis que la fonction de cette protéine est très conservée dans toutes les cellules eucaryotes [4]. En effet, Cdc42 contrôle une partie des transports intracellulaires essentiels à la polarisation des cellules épithéliales et l'orientation de la migration des neutrophiles en réponse à un gradient de substances chimiotactiques. Dans le modèle que nous avons mis au point, elle contrôle aussi la polarité des astrocytes lors de l'induction de leur migration en réponse à une lésion qui, in vitro, est une rayure de la monocouche cellulaire [5]. Dans ce modèle, Cdc42 agit via un de ses effecteurs, Par6, associé à une protéine kinase $C$ atypique (aPKC), PKC $\zeta$ (Figure I) $(\rightarrow)$. Par6 semble être un effecteur privilégié de $\mathrm{Cdc42}$. Chez C. elegans, son rôle dans la polarité cellulaire, et celui des aPKC associées, a été mis en évidence indépendamment de celui de Cdc42 au cours des divisions asymétriques de l'embryon. La position asymétrique du fuseau mitotique nécessite la présence de Par3-Par6-PKC3 au pôle antérieur du zygote (Figure 2); quant à $\mathrm{Cdc42}$, elle contrôle la localisation du complexe associé à Par6. Chez la drosophile, Par6 et les aPKC sont aussi impliquées dans la polarisation cellulaire au cours de la différenciation de l'ovocyte et des divisions asymétriques des neuroblastes. Le complexe protéique formé de $\mathrm{Cdc} 42$, Par3, Par6 et aPKC est conservé dans l'ensemble du règne animal, et son rôle ne se limite pas au contrôle des divisions asymétriques [6]. La localisation de ce complexe est très spécifique des cellules: dans les cellules épithéliales, on le trouve dans les jonctions cellulaires, et il joue un rôle crucial dans la mise en place de la polarité baso-apicale de ces cellules; dans les neurones, il est présent exclusivement dans le cône de croissance axonal et gouverne la mise en place et le maintien de la croissance axonale, essentielle à la polarité des neurones (Figure 2). Dans les astrocytes migrant en réponse à la lésion de la monocouche, l'activation de $\mathrm{Cdc} 42$ et le recrutement et l'activation du complexe Par6-PKC $\zeta$ se font au pôle antérieur des cellules (Figure2). La façon dont le complexe Cdc42-Par6-PKC $\zeta$ est recruté à l'avant des cellules reste toutefois un point encore à éclaircir. 


\section{Le contrôle de GSK3}

par le complexe Cdc42, Par6, PKC

Si le complexe Cdc42-Par6-aPKC est

essentiel à la polarité cellulaire toutes espèces confondues, il reste à comprendre comment ce complexe contrôle la polarité dans des contextes cellulaires variés, et quels sont ses liens avec les nombreuses autres protéines impliquées dans la polarité cellulaire.

Dans notre modèle astrocytaire, GSK3 $\beta$ s'est imposée comme un intermédiaire essentiel entre le complexe $\mathrm{Cdc} 42$, Par6, aPKC et la réorientation des microtubules [7]. Cette kinase est phosphorylée en réponse à la lésion de la monocouche, réaction contrôlée par Cdc42, Par6 et PKC $\zeta$; la forme phosphorylée de GSK3 $\beta$ est localisée avec Cdc42 et PKC $\zeta$ à l'avant des cellules en migration (Figure 2) [7]. Enfin, la phosphorylation de GSK3 $\beta$ est nécessaire à la réorientation du centrosome et du cytosquelette de microtubules (Figure 1). GSK3 $\beta$ a été impliquée dans la rotation du fuseau mitotique au cours du développement précoce de C. elegans, et son inhibition provoque des défauts dans l'alignement des chromosomes au cours de la mitose [8]. GSK3 $\beta$ est aussi essentielle à la polarisation de l'embryon de xénope et d'oursin [9] (Figure 2).

$A P C$, un gène suppresseur de tumeur impliqué dans la polarité cellulaire GSK3 $\beta$ peut agir sur les microtubules en phosphorylant des protéines qui leur sont associées comme MAPIB ou APC (adenomatous polyposis coli) [10]. Par son association avec l'axine, APC et la $\beta$-caténine, GSK3 $\beta$ est aussi un composant majeur de la voie de signalisation par Wnt et contrôle la dégradation de la $\beta$-caténine et la régulation de la trans$(\rightarrow) \mathrm{m} / \mathrm{s}$ cription [1]] $(\rightarrow)$. Dans le cas de la $2003, \mathrm{n}^{\circ} 2$, migration astrocytaire, cette voie de p. 187 cellules en migration (Figure I). L'expression d'une forme tronquée d'APC dépourvue des domaines de liaison aux microtubules, fréquente dans les cas génétiques de cancer colorectal [12], inhibe la réorientation du centrosome. Cette forme tronquée d'APC pourrait donc contribuer à la formation de tumeurs colorectales par deux mécanismes: d'une part, une augmentation de la transcription (voie de signalisation de type Wnt) conduisant à une augmentation des divisions cellulaires, d'autre part, l'induction d'une perte de la polarité des cellules épithéliales de l'intestin, source d'instabilité génétique au cours des divisions cellulaires. L'étude de la migration astrocytaire a donc mis en évidence un lien entre deux voies de signalisation conservées, $\mathrm{Cdc42-}$ Par6-aPKC et GSK3-APC, qui convergent pour établir la polarité cellulaire. Quant au caractère systématique de ce lien dans l'établissement de la polarité cellulaire, il devra être testé dans d'autres modèles cellulaires. Le rôle de Par6 et des aPKC vient d'être démontré lors de la mise en place de la polarité neuronale [13], et celui de GSK3 est déjà reconnu comme essentiel dans la migration du cône de croissance axonal [14]. Dans ce modèle, la forme phosphorylée de GSK3 et la zone d'accumulation de Par6 sont localisées à l'extrémité du cône de croissance (Figure 2), ce qui suggère, dans cet exemple aussi, la convergence des deux voies de signalisation.

La conservation de ces voies de signalisation démontre le rôle fondamental dans la vie animale des protéines impliquées dans la polarité. Ces molécules sont nécessaires non seulement au développement, mais aussi aux fonctions de nombreuses cellules différenciées. II n'est donc pas étonnant que certaines, comme APC, agissent comme suppresseur de tumeur. La perte de la polarité cellulaire pourrait être un élément déterminant de la formation de cellules tumorales. $\diamond$

A crucial protein complex for cell polarity

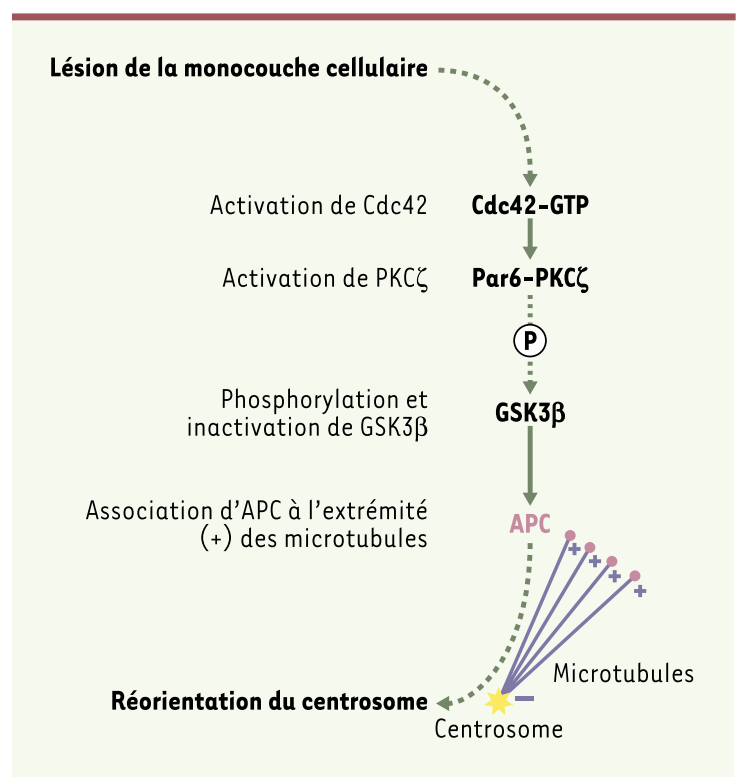

Figure 1. Voie de signalisation contrôlant la polarisation des astrocytes pendant leur migration. La lésion de la monocouche astrocytaire cause l'activation de la petite protéine $\mathrm{G} C \mathrm{dc} 42$ et, en conséquence, le recrutement et l'activation du complexe

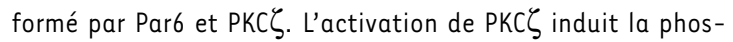
phorylation de GSK3 $\beta$ (glycogen synthase kinase $3 \beta$ ) et la relocalisation d'APC (adenomatous polyposis coli), qui s'associe alors à l'extrémité (+) des microtubules. Cette voie de signalisation est nécessaire à la réorientation des microtubules et $\mathrm{du}$ centrosome dans la direction de la migration. 


\section{RÉFÉRENCES}

1. Stowers L, Yelon D, Berg LJ, Chant J. Regulation of the polarization of T cells towards antigenpresenting cells by Rasrelated GTPase CDC42. Proc Natl Acad Sci USA 1995; 92: 5027-31.

2. Nobes $C D$, Hall A. Rho GTPases control polarity, protrusion and adhesion during cell movement. J Cell Biol 1999; 144: 1235-44.

3. Johnson DJ. Cdc42: an essential Rho-type GTPase controlling eukariotic cell polarity. Microbiol Mol Biol Rev 1999; 63: 54-105.

4. Etienne-Manneville $S$, Hall A. Rho GTPases in cell biology. Nature 2002; 420: 629-35.

5. Etienne-Manneville S. Les molécules qui dirigent la migration des astrocytes. Med Sci 2002; 18: 142-4.
6. Etienne-Manneville $S$, Hall A. Cell polarity: Par6, aPKC and cytoskeletal crosstalk. Curr Opin Cell Biol 2003; 15: 67-72.

7. Etienne-Manneville $S$, Hall A. Cdc42 regulates GSK3 and adenomatous polyposis coli (APC) to control cell polarity. Nature 2003; 420: 629-35.

8. Schlesinger A, Shelton CA, Maloof JN, Meneghini M, Bowerman B. Wnt pathway components orient a mitotic spindle in the early Caenorhabditis elegans embryo without requiring gene transcription in the responding cell. Genes Dev 1999; 13: 2028-38.

9. Itoh K, Krupnik VE, Sokol Sy. Axis determination in Xenopus involves biochemical interactions of axin, glycogen synthase kinase 3 and $\beta$-catenin. Curr Biol 1998; 8: 591-4.
10. Zumbrunn J, Kinoshita K, Hyman AA, Nathke IS. Binding of the adenomatous polyposis coli protein to microtubules increases microtubule stability and is regulated by GSK3 beta phosphorylation. Curr Biol 2001; 11: 44-9.

11. Rubinfeld B, Albert I, Porfiri $\varepsilon$, Fiol C, Munemitsu S, Polakis P. Binding of GSK3beta to the APC-betacatenin complex and regulation of complex assembly. Science 1996; 272: 1023-6.

12. Polakis P. Wnt signaling and cancer. Genes Dev 2000; 14: 1837-51.
13. Shi SH, Jan Ly, Jan YN. Hippocampal neuronal polarity specified by spacially localized $\mathrm{mPar} 3 / \mathrm{mPar6}$ and PI3kinase activity. Cell 2003; 112: 63-75.

14. Eickholt BJ, Walsh FS, Doherty P. An inactive pool of GSK-3 at the leading edge of growth cones is implicated in Semaphorin 3A signaling. J Cell Biol 2002; 157: 211-7.

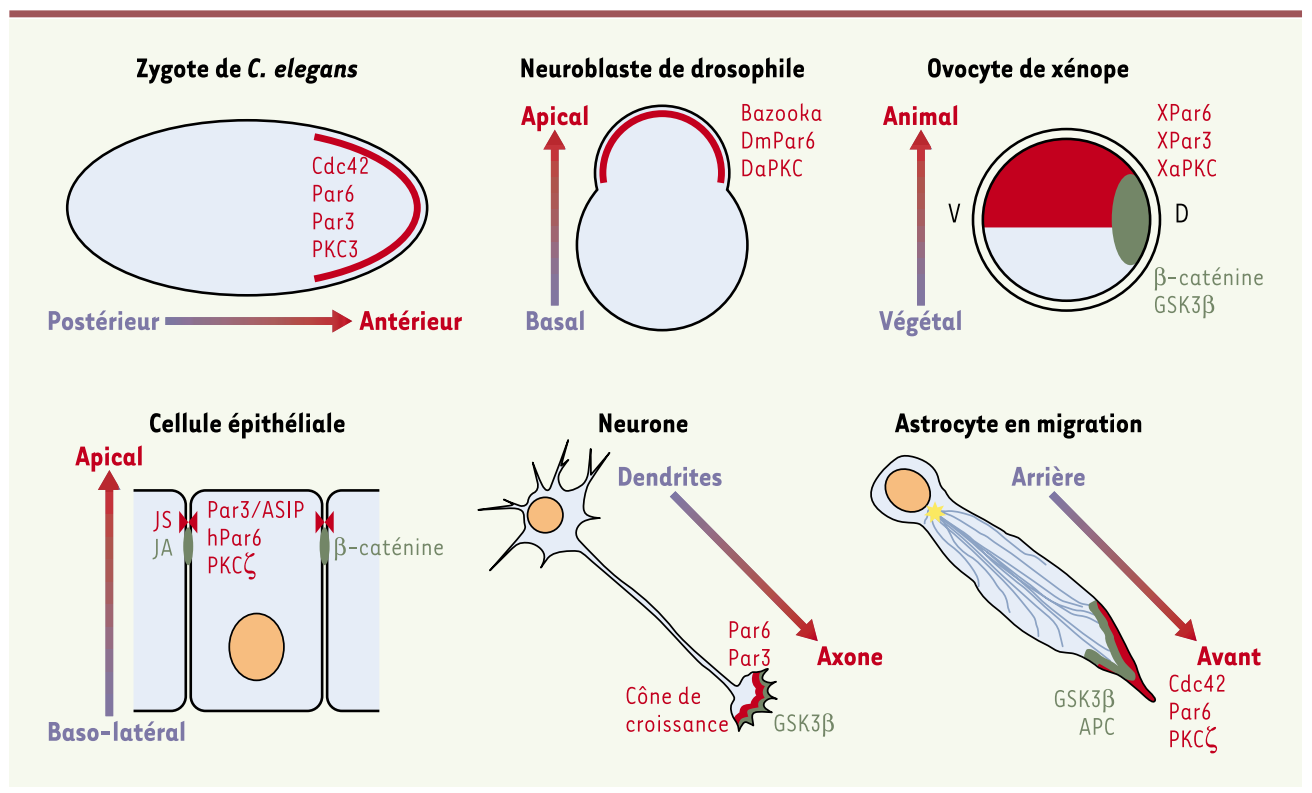

Figure 2. Conservation des molécules contrôlant la polarisation cellulaire. Différents modèles d'étude ont mis en évidence le rôle des protéines Cdc42, Par6, Par3 et aPKC (en rouge), d'une part, et de GSK3 $\beta$, $\beta$-caténine et APC (en vert), d'autre part, dans la polarité cellulaire. Par3, Par6, PKC3 et Cdc42 contrôlent les divisions asymétriques de l'embryon de $C$. elegans, et les orthologues de ces protéines sont impliqués dans les divisions asymétriques des neuroblastes de drosophile. Chez les vertébrés, les orthologues de ces mêmes protéines sont aussi impliqués dans la polarisation de cellules différenciées comme les cellules épithéliales, les neurones et les astrocytes en migration. Dans tous ces cas, la localisation précise de ces protéines est essentielle à leur fonction. GSK3 $\beta$, ainsi que ses substrats $\beta$-caténine et APC, sont essentiels au contrôle de la polarité de l'œuf de xénope et des astrocytes en migration. Là encore, la localisation de ces protéines est contrôlée de façon précise. 